\section{Digital gangrene: an unusual cause}

\section{A Sharma, M E Yeolekar, N K Hase, N Ashwini}

Answers on $p 121$.

A 27 year old man, with no addictions, was admitted with a history of fever and blackening of his right little finger of one day's duration. There was no history of trauma or joint pains, and no previous symptoms of any other cardiac illness in the past. The fever was high grade associated with chills; there were no episodes of chest pain, dyspnoea, loose motions, or pain in the abdomen.

Physical examination revealed an acutely ill young man who was conscious oriented and alert. On examination his pulse was 110 beats/min, regular in volume with no apex pulse deficit, and blood pressure was 90/ $60 \mathrm{~mm} \mathrm{Hg}$. On examination of his extremities we found that there was an erythematous macular rash on the ulnar aspect of his right hand; a similar rash was also found on his right foot at the

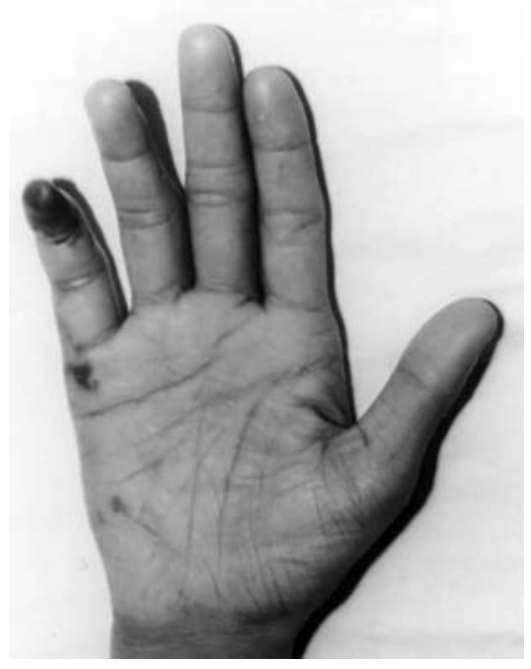

Figure 1 Patient's finger.

Figure 2 TOE of the patient. big toe. His right little finger showed evidence of dry gangrene with a line of demarcation between the normal and the gangrenous area (fig 1). All peripheral pulses were well felt. The patient also had grade 2 clubbing of all the digits. On examination of his cardiovascular system there was a systolic murmur at the aortic area; there was no other thrill or murmur at other areas. On respiratory system examination there were crepts present in the bases of both the lungs. Examination of the central nervous system did not reveal any focal neurological deficit.

Laboratory investigation revealed a haemoglobin concentration of $102 \mathrm{~g} / \mathrm{l}$, white cell count of $17 \times 10^{9} / 1$ with $90 \%$ neutrophils; his erythrocyte sedimentation rate was $100 \mathrm{~mm}$ after one hour. and urine examination did not show haematuria.

In view of the skin changes and the murmur serial blood cultures were done. As the initial two dimensional echocardiography did not show any vegetation transoesophageal echocardiography (TOE; fig 2) was carried out; electrocardiography gave normal results.

\section{QUESTIONS}

(1) What are the cutaneous changes in the hand?

(2) What did the blood culture grow and what is the antibiotic of choice?

(3) What does the TOE show?

Postgrad Med J 2004;80:1 18.

doi: 10.1136/pmj.2003.005892q

\section{$\cdots \cdots \cdots \cdots \cdots$}

\section{Authors' affiliations}

A Sharma, M E Yeolekar, N K Hase,

N Ashwini, Department of Internal Medicine,

LTMG Hospital, Sion, Mumbai, India

Correspondence to: Dr Anand Sharma, 15/2 Shivpuri, Chembur Naka, Mumbai 400 071, India; drandy2003@yahoo.co.in

Submitted 28 January 2003

Accepted 22 March 2003

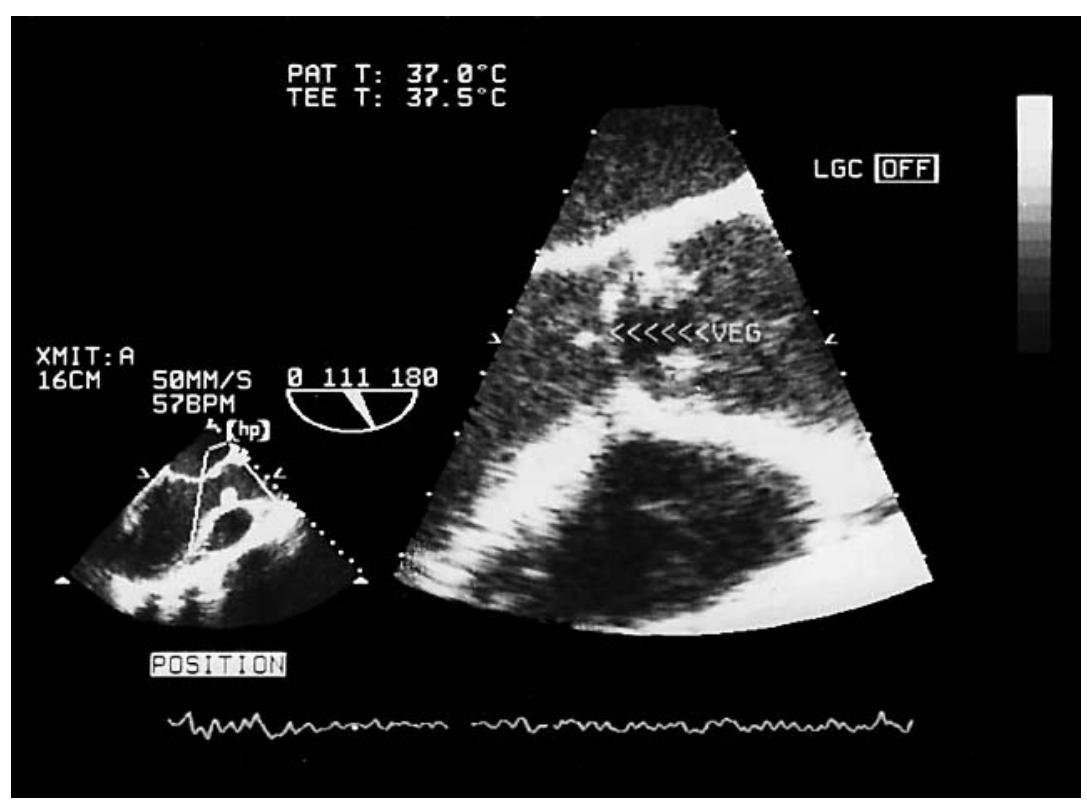

